\title{
Autosomal dominant striatal neurodegeneration
}

INSERM

\section{Source}

INSERM. (1999). Orphanet: an online rare disease and orphan drug data base. Autosomal dominant striatal neurodegeneration. ORPHA:228169

Autosomal dominant striatal degeneration (ADSD) is an adult-onset movement disorder characterized by bradykinesia, dysarthria and muscle rigidity. 